\title{
PROJETO DE CONTROLADOR POR ALOCAÇÃO DE POLOS APLICADO A UM SISTEMA DE SUSPENSÃO ATIVA
}

\author{
$\underline{\text { Joacy Mesquita da Silva }}{ }^{1}$; Márcia Lissandra Machado Prado ${ }^{2}$ \\ 1. Bolsista PROBIC/UEFS, Graduando em Engenharia de Computação, Universidade Estadual de Feira de Santana, \\ e-mail: joacymsilva@gmail.com \\ 2. Orientadora, Departamento de Tecnologia, Universidade Estadual de Feira de Santana, e-mail: \\ marcia.lissandra@gmail.com
}

PALAVRAS-CHAVE: Teoria de Controle, Alocação de Polos, Sistema de Suspensão Ativa.

\section{INTRODUÇÃO}

Os sistemas de controle são importantes em diversos campos da engenharia e da ciência, eles são utilizados nas mais diversas áreas da indústria (Ogata, 2009). Em veículos espaciais, sistemas robóticos, e operações industriais que envolvam o controle de temperatura, vazão, pressão, estabilização, direção, estão exemplos de sua utilização (Nise, 2009).

A Teoria de Controle Robusto estuda métodos para a construção de controladores com o propósito de melhorar significativamente o desempenho de sistemas de controle complexos, aplicáveis especialmente à indústria (Almeida, 2013). Quando, no projeto do controlador, há o desejo de especular a existência de erros ou incertezas entre a planta real e o seu modelo matemático, tal teoria é empregada (Ogata, 2009).

As suspensões veiculares têm por finalidade suportar, de forma adequada, o chassi do veículo, fazendo isolamento das vibrações causadas por irregularidades do terreno; manter o contato dos pneus com o solo de modo a manter o carro o mais estável possível; e proporcionar melhor dirigibilidade e conforto aos seus ocupantes (Côrte-real, 2002; Silva; Grandinetti, 2005).

Este trabalho teve por objetivo descrever o projeto de um controlador robusto por alocação de polos aplicado a um Sistema de Suspensão Ativa. Como não foi possível fazer o projeto do controlador robusto, será apresentado o projeto do controlador por alocação de polos aplicado a esse Sistema.

\section{METODOLOGIA}

Para a pesquisa, foi utilizado o Sistema de Suspensão Ativa da Quanser. O modelo físico concedido pelo fabricante se encontra na Figura 1.

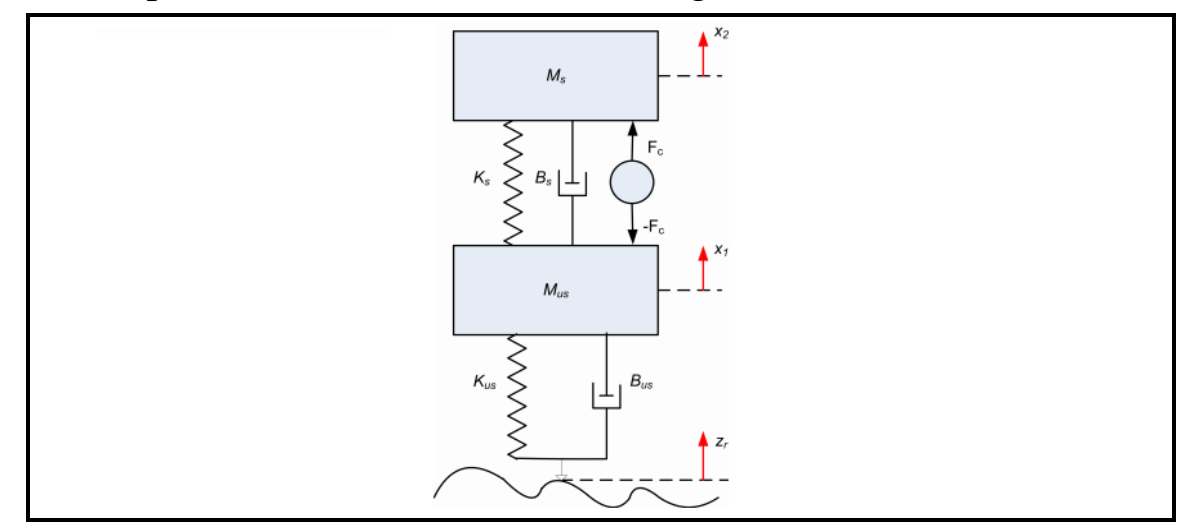

Figura 1. Modelo para o Sistema de Suspensão Ativa da Quanser (QUANSER, 2013) 
O Sistema consiste em duas massas móveis suportadas por um par de molas e amortecedores. A massa superior corresponde ao corpo do veículo suportado acima da suspensão, enquanto a massa inferior se refere a uma das rodas do veículo. A ondulação representa a pista. A modelagem matemática foi possível através da representação do sistema em Equações Diferenciais, as equações 1 e 2 foram obtidas.

$$
\begin{gathered}
\frac{d^{2} x_{2}}{d t^{2}}=-g+\frac{F_{c}}{M_{s}}+\frac{B_{s}}{M_{s}} \frac{d x_{1}}{d t}-\frac{B_{s}}{M_{s}} \frac{d x_{2}}{d t}+\frac{K_{s} x_{1}}{M_{s}}-\frac{K_{s} x_{2}}{M_{s}}(1) \\
\frac{d^{2} x_{1}}{d t^{2}}=-g-\frac{F_{c}}{M_{u s}}-\frac{\left(B_{s}+B_{u s}\right)}{M_{u s}} \frac{d x_{1}}{d t}+\frac{B_{u s}}{M_{u s}} \frac{d x_{2}}{d t}+\frac{B_{u s}}{M_{u s}} \frac{d z_{r}}{d t}-\frac{\left(K_{s}+K_{u s}\right) x_{1}}{M_{u s}}+\frac{K_{s} x_{2}}{M_{u s}}+\frac{K_{u s} Z r}{M_{u s}}(2)
\end{gathered}
$$

O sistema é considerado do tipo MISO (Multiple Input, Single Output), possui como entradas a força de controle $\boldsymbol{f}_{\boldsymbol{c}}$ e a perturbação $\boldsymbol{z}_{r}$, que representa as ondulações da pista, a força de controle deve ser capaz de minimizar os efeitos causados pela ação da perturbação. A variável a ser controlada é $\boldsymbol{x}_{\mathbf{2}}$, que diz respeito à posição da carroceria do veículo. A variável $\boldsymbol{x}_{\mathbf{1}}$ diz respeito à posição do pneu. A aceleração da gravidade é representada pela constante $\boldsymbol{g}$, os efeitos da força da gravidade podem ser eliminados, considerando pontos de equilíbrio do sistema. A saída considerada, variável a ser controlada, passa a ser chamada de $\boldsymbol{z}_{\boldsymbol{s}}$. Os valores numéricos atribuídos a cada parâmetro da Figura 1 são apresentados na Tabela 1.

Tabela 1. Parâmetros do Sistema de Suspensão Ativa

\begin{tabular}{|c|c|c|}
\hline Parâmetro & Identificação & Valor \\
\hline $\boldsymbol{M}_{\boldsymbol{s}}$ & Massa do carro & $2,45 \mathrm{Kg}$ \\
\hline $\boldsymbol{M}_{\boldsymbol{u}}$ & Massa do pneu & $1 \mathrm{Kg}$ \\
\hline $\boldsymbol{K}_{\boldsymbol{s}}$ & Constante de rigidez da suspensão & $900 \mathrm{~N} / \mathrm{m}$ \\
\hline $\boldsymbol{K}_{\boldsymbol{u}}$ & Constante de rigidez do pneu & $2500 \mathrm{~N} / \mathrm{m}$ \\
\hline $\boldsymbol{B}_{\boldsymbol{s}}$ & Coeficiente da suspensão & $7,5 \mathrm{~N} . \mathrm{s} / \mathrm{m}$ \\
\hline $\boldsymbol{B}_{\boldsymbol{u}}$ & Coeficiente do pneu & $5 \mathrm{~N} . \mathrm{s} / \mathrm{m}$ \\
\hline
\end{tabular}

Aplicando a Transformada de Laplace podemos obter a resposta em frequência do sistema, ou seja, a representação do mesmo na forma de Função de Transferência. Foram obtidas duas funções, uma relaciona a saída $\boldsymbol{z}_{\boldsymbol{s}}$ e a força de controle $\boldsymbol{F}_{\boldsymbol{c}}$, chamada de $G_{1}(s)$, equação 3 , e a outra relaciona a saída $\boldsymbol{z}_{\boldsymbol{s}}$ e a perturbação $\boldsymbol{z}_{\boldsymbol{r}}$, chamada de $G_{2}(s)$, equação 4. A saída total é obtida pela soma da interação das duas entradas com suas respectivas funções de transferência.

$$
\begin{aligned}
& G_{1}(s)=\frac{Z_{s}(s)}{F_{c(S)}}=\frac{\mathrm{s}^{2}+7.5 \mathrm{~s}+1250}{2.45 \mathrm{~s}^{4}+38.125 \mathrm{~s}^{3}+6205 \mathrm{~s}^{2}+13875 \mathrm{~s}+1.125 e 06} \\
& G_{2}(s)=\frac{Z_{S}(s)}{Z_{r(s)}}=\frac{37.5 \mathrm{~s}^{2}+13875 \mathrm{~s}+1.125 e 06}{2.45 \mathrm{~s}^{4}+38.125 \mathrm{~s}^{3}+6205 \mathrm{~s}^{2}+13875 \mathrm{~s}+1.125 e 06}
\end{aligned}
$$

Para entender o comportamento do sistema, foi desenvolvida a simulação no Simulink, a Figura 2 nos traz a resposta natural do sistema em Malha Fechada. Por indicação do fabricante, a perturbação $\boldsymbol{z}_{\boldsymbol{r}}$ é representada por uma onda quadrada de amplitude $0,01 \mathrm{~m}$ e frequência $0,3 \mathrm{~Hz}$. 


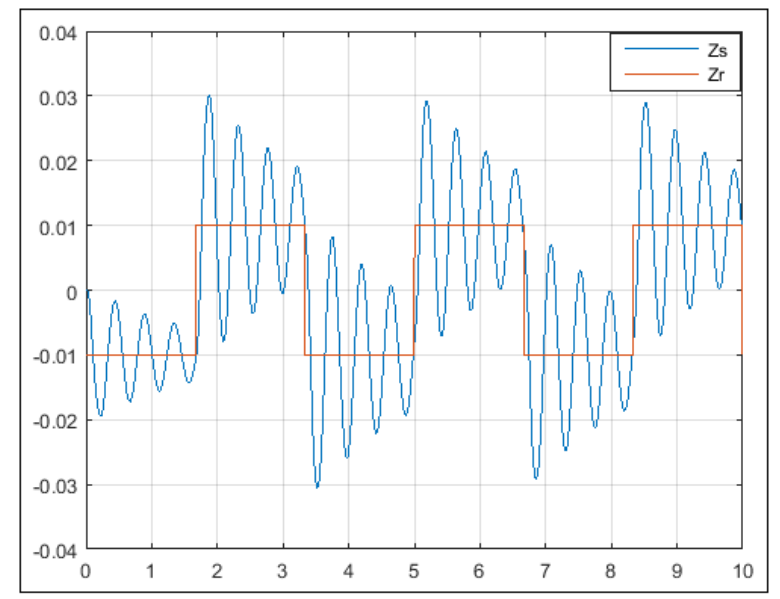

Figura 2. Resposta do Sistema em Malha Fechada.

Através da visualização da Figura 2, verificamos que a saída apresenta muita oscilação. A planta possui um atuador responsável por aplicar a força de controle Fc, o objetivo é encontrar a equação para o controlador, $G_{C}(s)$, de modo a minimizar as ondulações geradas pelo efeito da perturbação Zr. A Figura 3 mostra a disposição dos blocos do sistema controlado.

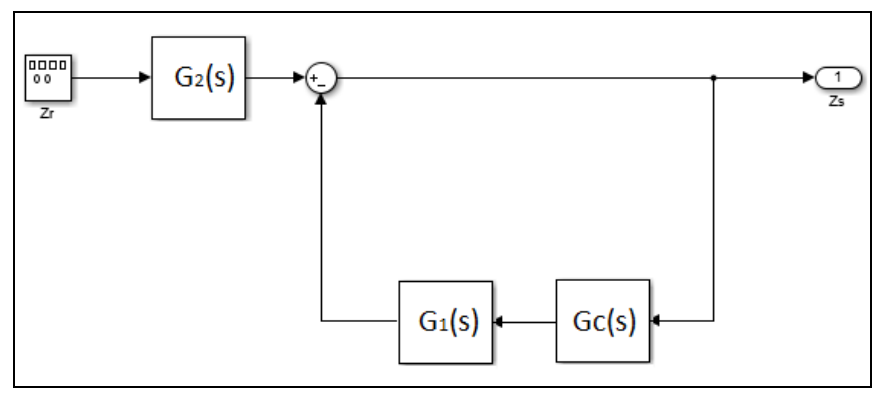

Figura 3. Diagrama de Blocos do Sistema controlado.

Foram feitas diversas tentativas com variados tipos de controlador, podendo ser destacado $G_{C}(s)=\frac{A s^{3}+B s^{2}+C s+D}{E s+F}$. Foi utilizado controle por Alocação de Polos para encontrar os coeficientes do controlador, o método consiste em escolher o posicionamento dos pólos da equação de malha fechada do sistema, e a partir disso, encontrar a equação do denominador de malha fechada, para posteriormente relacionála com os coeficientes a serem encontrados para o controlador. A equação de malha fechada é apresentada na equação 5.

$$
T(s)=G_{2}(s) \frac{1}{1+G_{1}(s) G_{c}(s)}(5)
$$

\section{RESULTADOS E/OU DISCUSSÃO}

Para o projeto utilizando como controlador $G_{C}(s)$, os pólos foram alocados com o intuito de eliminar o efeito dos zeros. Foram alocados polos em $s=-250, s=$ $-120, s=-20, s=-7.1720+j 47.6002$, e $s=-7.1720-j 47.6002$. Com isso o denominador da função de transferência de malha fechada pode ser encontrado: $\operatorname{den} T(s)=\mathrm{s}^{5}+404.344 \mathrm{~s}^{4}+4.5311 e 04 \mathrm{~s}^{3}+2.04018 e 06 \mathrm{~s}^{2}+9.52703 e 07 \mathrm{~s}+1.3903 e 09$.

Conhecendo o denominador de malha fechada, foi possível encontrar os coeficientes do controlador. Foram obtidos $A=31.9604, \quad B=993.6712, \quad C=8.1724 e 04$, $D=1.2398 e 06, E=-12.6369$, e $F=-141.7084$. A equação 6 se refere a $G_{C}(s)$.

$$
G_{C}(s)=\frac{31.9604 \mathrm{~s}^{3}+993.6712 \mathrm{~s}^{2}+8.1724 e 04 \mathrm{~s}+1.2398 e 06}{-12.6369 \mathrm{~s}-141.7084}(6)
$$


Conhecendo o controlador e a disposição dos blocos em Malha Fechada, a resposta do sistema controlado pode ser obtida no Matlab. A Figura 3 mostra o resultado dessa simulação.

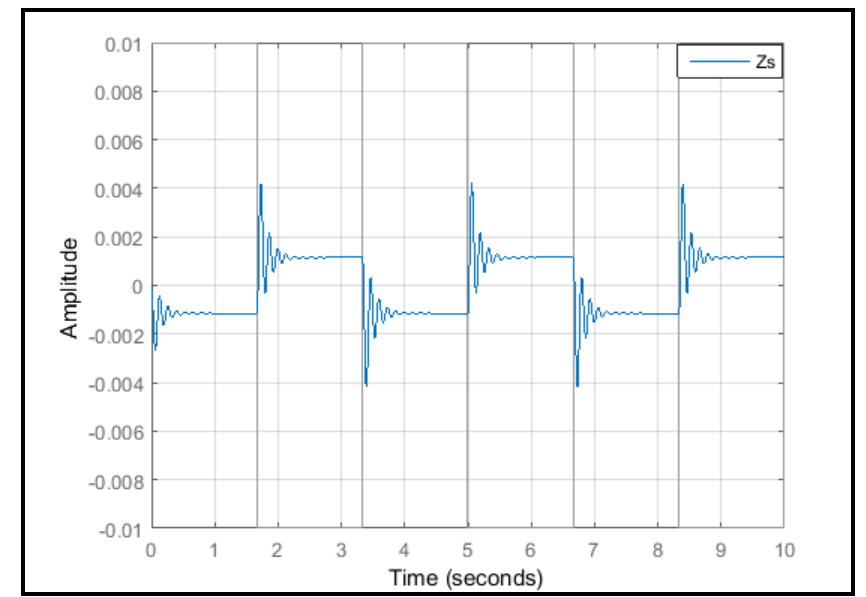

Figura 3. Resposta do Sistema em Malha Fechada, com a ação do Controlador

Comparando com a resposta em Malha Fechada sem ação do controlador, podemos verificar que o sistema controlado apresentou considerável diminuição na amplitude das oscilações. Além disso, pode ser percebida a minimização dos efeitos da perturbação $\mathbf{z}_{\mathbf{r}}$ na carroceria do veículo.

\section{CONSIDERAÇÕES FINAIS}

Neste trabalho puderam ser aplicadas técnicas de controle por Alocação de Polos para melhorar o desempenho do Sistema de Suspensão Ativa da Quanser. Comparando com o sistema sem nenhum controlador, o sistema controlado se mostrou eficaz na minimização dos efeitos causados pela interação da perturbação com o sistema, gerando também estabilidade na resposta.

Como os resultados satisfatórios foram obtidos no fim da duração do projeto, não foi possível aplicar os conceitos de Controle Robusto e Análise Intervalar adquiridos durante a revisão de literatura. Pretende-se dar seguimento ao trabalho, nos aspectos citados anteriormente e na verificação do esforço de controle do sistema.

\section{REFERENCIAS}

AGUIRRE, L. A. (2007). Enciclopédia de Automática: Controle e Automação. $1^{\mathrm{a}}$ Edição. São Paulo: Blucher.

ALMEIDA, K. S., PRADO, M. L. M. (2013).Projeto de um controlador robusto e ótimo para um sistema de suspensão ativa. In: Seminário de Iniciação Científica - UEFS, 2013, Feira de Santana. Anais do Semic.

CÔRTE-REAL, E. W. (2002). Dessensibilização da Estrutura de Controle LQG Aplicada ao Modelo de uma Suspensão Ativa Utilizando a Técnica PRABI. Rio de Janeiro: Instituto Militar de Engenharia, 2002. Disponível em: <http://www.pgee.ime.eb.br/pdf/edilmar_real.pdf>. Acesso em: 30 mar. de 2016.

FRANKLIN, G. F., POWELL, J. D. e EMANI-NAEINI, A. (2009). Feedback Control of Dynamic Systems. 6a Edição. New Jersey, Pearson.

NISE, N. S. (2009). Engenharia de Sistemas de Controle. 5a Edição, Rio de Janeiro, LTC.

OGATA, K. (2009). Engenharia de Controle Moderno, 4a Edição, Prentice Hall. 